\title{
Numerical Solution for First Kind Fredholm Integral Equations by Using Sinc Collocation Method
}

\author{
Khosrow Maleknejad ${ }^{1 *}$, Yaser Rostami ${ }^{2}$, Hamed Shahi Kalalagh ${ }^{3}$ \\ 1 Department of Mathematics, Fandanesh Institute of Higher Education, Saveh, Iran. \\ 2 Department of Mathematics, Karaj Branch, Islamic Azad University, Karaj, Iran. \\ 3 Department of Mathematics, Iran University of Science and Technology, Narmak, Tehran, Iran. \\ * Corresponding author. Email: maleknejad@iust.ac.ir \\ Manuscript submitted February 4, 2016; accepted June 12, 2016. \\ doi: 10.17706/ijapm.2016.6.3.120-128
}

\begin{abstract}
In this paper, we proposed an advanced numerical model in solving the Fredholm integral equations of the first kind, by using Sinc basis functions. This method has been shown to be a powerful numerical solution for finding accurate solutions. So, in this paper, some properties of the Sinc-collocation method are used to reduce integral equation of the first kind to some algebraic equations. Numerical results are included to verify the accuracy of this method. 2010 Mathematics Subject Classification: 45B05; 65R20.
\end{abstract}

Key words: Fredholm integral equations, Sinc approximation, regularity.

\section{Introduction}

The Integral equations play an important role in many fields of science and engineering. The most useful integral equations are Fredholm equation. This equations have been used in many fields [1], such as control, economics, electrical engineering, medicine, etc. In recent years, many diferent orthonormal basis functions, such as Fourier functions, wavelets [2], sinc functions [3], [4], etc have been used to estimate the solution of these integral equations. However, the most attractive one among them especially for solving linear and nonlinear integral equations may be the sinc functions as basis [5], [6]. Sinc functions were used for solving integral equations which they produced some good approximations [7],[8].

In some papers such as integral equation of first kind (FK1) have been discussed by some authors, but in these papers the method of regularization is not mentioned. In the present article, we are concerned the application of sinc-collocations method (SC) to find the approximate solution of Fredholm integral equation of first kind:

$$
\int_{a}^{b} k(x, s) \varphi(s) d s=f(x),-\infty<a \leq x \leq b<\infty,
$$

where $k(x, s), f(x)$ are given functions and $\phi(s)$ is an unknown function to be determined.

This type of equations have seen in many science and engineering fields, and in many cases, we can not solve this equation analytically [9], [10]. There are several advantages to using approximations with sinc functions as basis. Because of their rapid convergence, Sinc numerical methods do not suffer from the common instability problems which have been observed in other numerical methods [11]-[13]. 
This paper is organized as follows. In Section 2, we introduce the cardinal function of sinc method. Section 3 (SC) method are obtained by means of the Sinc approximation. In Section 4, we discuss the convergence of numerical method applied for (1). In Section 5, numerical results will be given to examine the efficient of the proposed methods. Finally in Section 6 we conclude this paper.

\section{Sinc Function and Basic Definition}

\subsection{Sinc Interpolation}

The Sinc function is defined on the whole real line by [14]

$$
\operatorname{Sinc}(x)=\left\{\begin{array}{cc}
\frac{\sin (\pi x)}{\pi x} & x \neq 0 \\
1 & x=0
\end{array}\right.
$$

Now, for $h>0$ and integer $k$, we define kth Sinc function with step size $h$ by

$$
S(k, h)(x)=\frac{\sin (\pi(x-k h) / h)}{\pi(x-k h) / h} .
$$

\subsection{Sinc Approximation on Real Line}

$\phi$ is approximated by using the finite number of terms in (2). For positive integer $N$, we defined

$$
\varphi(x) \approx \sum_{k=-N}^{N} \varphi(k h) S(k, h)(x) .
$$

For purpose of explanation of the procedure, we consider the following definitions in [8].

\subsubsection{Definition}

Let $D$ be a simply-connected domain in the complex plane having boundary $\partial D$ and $H^{1}\left(D_{d}\right)$ denote the family of all functions $F$ that are analytic in $D_{d}$, such that $D_{d}$ defined by

$$
D_{d}=\{z \in C:|\operatorname{Im}(z)|<d\}
$$

such that for $0<\varepsilon<1, D_{d}$ is defined by

$$
D_{d}(\varepsilon)=\left\{z \in C:|\operatorname{Im}(z)|<d(1-\varepsilon),|\operatorname{Re}(z)|<\frac{1}{\varepsilon}\right\},
$$

then

$$
N\left(F, D_{d}\right)=\lim _{\varepsilon \rightarrow 0}\left(\int_{\partial D_{d}}|F(z) d z|\right)<\infty .
$$

\subsection{Sinc Approximation on Interval $[a, b]$}


Let $\mu(z)$ denote a conformal map which maps the simply connected domain $D$ with boundary $\partial D$ onto a strip region $D_{d}$ So that on $\tau=[a, b]$ are map to $(-\infty, \infty)$ and we denote $\tau_{a}=a$ and $\tau_{b}=b$ then approximate solution (3) associated with the conditions [4].

$$
\lim _{x \rightarrow \tau_{a}} \varphi(x)=\lim _{x \rightarrow \tau_{b}} \varphi(x)=0 .
$$

Let $\varphi=\mu^{-1}$ denote the inverse map, now on a finite interval $[a, b]$ sinc approximation is defined as follow:

$$
\mu(x)=\ln \left(\frac{x-a}{b-x}\right) .
$$

which carries the eye-shaped complex domain

$$
\left\{z=x+i y:\left|\arg \left(\frac{z-a}{b-z}\right)\right|<d \leq \frac{\pi}{2}\right\},
$$

onto the infinite strip

$$
D_{d}=\left\{\omega=\alpha+\beta i:|\beta|<d<\frac{\pi}{2}\right\}
$$

On finite interval $[a, b]$ the basis functions are given by

$$
S(k, h) \circ \mu(x)=\frac{\sin (\pi(\mu(x)-k h / h))}{\pi(\mu(x)-k h / h)},
$$

now at points $x_{k}=k h$ Sinc function for interpolationx is followed by

$$
S(k, h)(j h)=\delta_{k j}^{(0)}= \begin{cases}1 & k=j \\ 0 & k \neq j\end{cases}
$$

So, $S(k, h) \circ \mu(x)$ shows Kronecker delta behavior on the grid points

$$
x_{k}=\phi(x)=\frac{a+b e^{k h}}{1+e^{k h}},
$$

and also approximation with sinc function for $\phi(x)$ over interval $[a, b]$ are

$$
\begin{aligned}
& \varphi(x) \approx \sum_{k=-N}^{N} \varphi\left(x_{k}\right) S(k, h) \circ \mu(x), \\
& \int_{a}^{b} \mu(x) d x \approx h \sum_{k=-N}^{N} \frac{\varphi\left(x_{k}\right)}{\mu^{\prime}\left(x_{k}\right)} .
\end{aligned}
$$




\section{Sinc-Collocation Method}

\subsection{The Method of Regularization}

Phillips (1962) develops a method for solving FKl by using the quadrature rule together with a regularization technique; later Twomey (1963) improved this method. It was Tikhonov (1963) who initially put forward a generalized theory. Assuming that a solution exists to the linear ill-posed problem FK1 (1) which can always be written in the generic form $K \phi=f$. If in (1) both $\phi(x), f(x) \in L_{2}$, and the kernel $k(x, s)$ is square-integrable, symmetric, and positive-definite, under these conditions we modify (1) and consider

$$
\int_{a}^{b} k(x, s) \varphi(s) d s+\gamma \varphi(x)=f(x),
$$

where $\gamma$ is known as the regularization parameter and (5) is an Fredholm integral equation of second kind (FK2) whose solution, denoted by $\phi_{\gamma}(x)$, can be found. These equations may be written as $\phi(K+\gamma I)=f$.

Substituting $\phi_{\gamma}(x)$ for $\phi(x)$ in (1), we get

$$
\int_{a}^{b} k(x, s) \varphi_{\gamma}(s) d s=f_{\gamma}(x)
$$

If $\left\|f(x)-f_{\gamma}(x)\right\| \leq \delta$ where $\delta$ is a preassigned quantity representing the tolerance of error, then the function $\phi_{\gamma}(x)$ is considered an acceptable approximate solution to (1).

\subsection{Approximation with Sinc Function}

In this section, we introduce the numerical solution for solving FK1 and to evaluate the unknown coefficients the properties of the Sinc function are used [12]-[13]. then we have (1). With (SC) method we can approximate unknown coefficients in expansion. If we let $N$ be a positive integer, then $\phi(x)$ which defined over the interval $[a, b]$, is approximated by using the sinc function as:

$$
\varphi(x)=\sum_{k=-N}^{N} \varphi\left(\mu^{-1}(k h) S(k, h) \circ \mu(s)\right)
$$

We rewrite the (1) By substituting Sinc approximation expansion (7) of unknown function $\phi(s)$, we have:

$$
\int_{a}^{b} k(x, s)\left(\sum_{k=-N}^{N} \varphi\left(\mu^{-1}(k h) S(k, h) \circ \mu(s)\right)\right) d s+\gamma \sum_{k=-N}^{N} \varphi\left(\mu^{-1}(k h) S(k, h) \circ \mu(x)\right)=f(x) .
$$

now to use Collocation method methods we need to find residual function as follow

$$
r_{N}\left(x_{i}\right)=\int_{a}^{b} k(x, s)\left(\sum_{k=-N}^{N} \varphi\left(\mu^{-1}(k h) S(k, h) \circ \mu(s)\right)\right) d s+\gamma \sum_{k=-N}^{N} \varphi\left(\mu^{-1}(k h) S(k, h) \circ \mu(x)\right)-f(x) .
$$


By collocating residual function at $\mathrm{n}=2 \mathrm{~N}+1$ distinct collocation points in interval $[a, b]$ for $r_{N}\left(x_{i}\right)=0$ we have:

$$
x_{i}=\mu^{-1}(i h)=\frac{a+b e^{i h}}{1+e^{i h}} ; i=-N,-N+1, \ldots, N-1, N .
$$

now integral equation converted to algebric system. also for evaluating matrix elements of this system we have

$$
\int_{a}^{b} k\left(x_{i}, s\right) S(k, h) \circ \mu(s) d s \approx h \sum_{j=-N}^{N} \frac{\int_{a}^{b} k\left(x_{i}, s_{j}\right) S(k, h) \circ \mu(s)}{\mu^{\prime}\left(s_{j}\right)} .
$$

where

$$
s_{j}=\mu^{-1}(j h)=\frac{a+b e^{j h}}{1+e^{j h}} ; j=-N,-N+1, \ldots, N-1, N .
$$

\section{Convergence Analysis}

The convergence of the (SC) methods which are introduced in the previous sections is discussed in this section.

\subsection{Theorem 1}

Let $\alpha, \beta$ and $d$ as positive constants, that

$$
1 . \phi \in H^{1}\left(D_{d}\right)
$$

2. $\phi$ decays exponentially on the real line by

$$
|\varphi(x)| \leq \alpha \exp (-\beta|x|), \forall x \in \mathfrak{R},
$$

then we have

$$
\sup \left|\varphi(x)-\sum_{k=-N}^{N} \varphi(k h) S(k, h)(x)\right| \leq C\left(N e^{-(\pi d \beta N)}\right)^{1 / 2},
$$

for some constant $C$ and step size $h$ is taken as

$$
h=\left(\frac{\pi d}{\beta N}\right)^{1 / 2} .
$$

proof [14]. 


\subsection{Theorem2}

Let $N$ be a positive integer, and let $h$ be selected by the

$$
h=\left(\frac{\pi d}{\beta N}\right)^{1 / 2} .
$$

Then if transformation function $\mu^{-1}(\xi)$ satisfies assumptions 1 and 2 in Theorem 1 with some $\alpha, \beta$ and $d$ we have

$$
\sup _{a<x<b}\left|\varphi(x)-\sum_{k=-N}^{N} \varphi\left(\mu^{-1}(k h)\right) S(k, h) \circ \mu(x)\right| \leq C(N \exp -(\pi d \beta N))^{1 / 2} .
$$

Proof [12].

The convergence rate of the method is $O(\exp (-c \sqrt{N}))$ with some $c$. For the solution of $k \phi=f$ choose the regularization parameter so that the size of the residual $r(\gamma)=\left\|k \phi_{\gamma}-f\right\|$ is the same as the error level in the data and the vector $\phi$ of minimum norm satisfying the requirement

$$
\|k \varphi-f\| \leq \delta
$$

where $P$ is the projector of Hilbert space

$$
P(k+\gamma I) \varphi_{\gamma}=P f P\left(\gamma \varphi_{\gamma}\right)+P\left(k \varphi_{\gamma}\right)=P f k \varphi_{\gamma}=f-\gamma \phi_{\gamma}\left\|k \varphi_{\gamma}-f\right\|=\left\|\gamma \varphi_{\gamma}\right\|=\left\|P f-P k \varphi_{\gamma}\right\|,
$$

if $\gamma \rightarrow 0$ given:

$$
\lim _{\gamma \rightarrow 0^{+}} r(\gamma)=\left\|P f-P k \phi_{\gamma}\right\| \leq \delta,
$$

and

$$
\lim _{\gamma \rightarrow \infty} r(\gamma)=\|f\| \geq \delta
$$

\section{Numerical Examples}

In order to discuss the performance of the (SC) as done on Matlab. Our achieved results are tabulated in Tables 1-3, in these tables the error on the Sinc grid points is defined as

$$
e(N)=\max \left|\varphi\left(s_{j}\right)-P \varphi\left(s_{j}\right)\right|
$$

The solutions of the given examples are obtained for regular parametr $\gamma=0.01$ and $\gamma=0.001$ and for different values of $N$.

Example 1. Consider the Fredholm first kind integral equation 


$$
\int_{0}^{1}\left(x^{2}+s^{2}\right)^{\frac{1}{2}} \varphi(s) d s=\frac{1}{3}\left[\left(1+x^{2}\right)^{\frac{3}{2}}-x^{3}\right],
$$

with exact solution $\phi(x)=x$. The solution for $\phi(x)$ is obtained by the methods in Section 3 . The approximate solution is evaluated for $\gamma=0.01$, and $\gamma=0.001$ and different values of $N$. The maximum absolute errors in numerical solution of Example 1 are tabulated in Table 1. These results are compared by the method which has been prepared in [8].

Table 1. Error values for Example 1

\begin{tabular}{c|c|c|c}
\hline \hline$N$ & $\gamma=0.01$ & $\gamma=0.001$ & method of $[8]$ \\
\hline 5 & $3.9 \times 10^{-2}$ & $1.4 \times 10^{-2}$ & $3.8 \times 10^{-4}$ \\
10 & $7.5 \times 10^{-3}$ & $3.8 \times 10^{-3}$ & $1.5 \times 10^{-4}$ \\
15 & $2.0 \times 10^{-3}$ & $7.6 \times 10^{-4}$ & $8.4 \times 10^{-5}$ \\
20 & $3.2 \times 10^{-4}$ & $9.3 \times 10^{-5}$ & $5.8 \times 10^{-5}$ \\
25 & $4.4 \times 10^{-5}$ & $2.1 \times 10^{-5}$ & $4.7 \times 10^{-5}$ \\
30 & $8.7 \times 10^{-6}$ & $3.9 \times 10^{-6}$ & $4.3 \times 10^{-5}$ \\
35 & $2.3 \times 10^{-6}$ & $7.8 \times 10^{-7}$ & $3.1 \times 10^{-5}$ \\
\hline
\end{tabular}

Table 2. Error values for Example 2

\begin{tabular}{c|c|c|c}
\hline \hline$N$ & $\gamma=0.01$ & $\gamma=0.001$ & method of [15] \\
\hline 2 & $4.1 \times 10^{-2}$ & $2.1 \times 10^{-2}$ & $5.1 \times 10^{-1}$ \\
3 & $5.3 \times 10^{-2}$ & $6.3 \times 10^{-3}$ & $5.8 \times 10^{-2}$ \\
4 & $6.4 \times 10^{-3}$ & $3.1 \times 10^{-3}$ & $8.4 \times 10^{-1}$ \\
5 & $5.6 \times 10^{-4}$ & $2.7 \times 10^{-4}$ & $9.6 \times 10^{-3}$ \\
6 & $4.6 \times 10^{-4}$ & $2.2 \times 10^{-4}$ & $3.3 \times 10^{-4}$ \\
7 & $3.1 \times 10^{-5}$ & $1.4 \times 10^{-5}$ & $1.7 \times 10^{-4}$ \\
8 & $3.7 \times 10^{-5}$ & $1.9 \times 10^{-5}$ & $4.7 \times 10^{-4}$ \\
\hline \hline
\end{tabular}

Table 3. Error values for Example 3

\begin{tabular}{c|c|c|c}
\hline \hline$N$ & $\gamma=0.01$ & $\gamma=0.001$ & method of [7] \\
\hline 2 & $2.5 \times 10^{-2}$ & $3.4 \times 10^{-3}$ & $2.0 \times 10^{-9}$ \\
3 & $5.4 \times 10^{-3}$ & $6.2 \times 10^{-4}$ & $5.3 \times 10^{-5}$ \\
4 & $1.4 \times 10^{-3}$ & $4.9 \times 10^{-4}$ & $1.3 \times 10^{-4}$ \\
5 & $2.8 \times 10^{-4}$ & $4.7 \times 10^{-5}$ & $2.2 \times 10^{-4}$ \\
6 & $3.2 \times 10^{-5}$ & $8.7 \times 10^{-6}$ & $3.0 \times 10^{-4}$ \\
7 & $4.3 \times 10^{-6}$ & $1.1 \times 10^{-6}$ & $5.9 \times 10^{-4}$ \\
8 & $8.5 \times 10^{-7}$ & $4.2 \times 10^{-7}$ & $3.8 \times 10^{-4}$ \\
\hline \hline
\end{tabular}

Example 2. Consider the Fk1 integral equation

$$
\int_{0}^{1} \mathrm{e}^{x s} \varphi(s) d s=\frac{\mathrm{e}^{x+1}-1}{x+1},
$$

with exact solution $\phi(x)=\exp (x)$. The maximum absolute errors in numerical solution of Example 2 are tabulated in Table 2. These results are compared by the method which has been prepared in [15]. 
Example 3. Consider the Fk1 integral equation with exact solution $\phi(x)=x$.

$$
\int_{0}^{1} \sin (x s) \varphi(s) d s=\frac{\sin x-x \cos x}{x^{2}} .
$$

The maximum absolute errors in numerical solution of Example 3 are tabulated in Table 3. these results are compared by the method which has been prepared in [7].

\section{Conclusion}

This paper has introduced Sinc-collocation method for fredholm integral equations of first kind. By use of Sinc basis and Sinc grid points in collocation method we convert these kind of integral equations to a system of algebraic equations. Our numerical experiments shows the accuracy and reliability of the proposed method. Tables 1-3 shows that as $\mathrm{N}$ increases the errors are decreased more rapidly, then for obtaining the better results using large number $N$ is recommended.

\section{References}

[1] Wazwaz, A. M. (2011). Linear And Nonlinear Integral Equations: Methods And Applications. Springer.

[2] Maleknejad, K., Sohrabi, S., \& Rostami, Y.( 2007). Numerical solution of nonlinear volterra integral equations of the second kind by using chebyshev polynomials. Journal of Applied Mathematics and Computation, 188, 123-128.

[3] Maleknejad, K., \& Nedaiasl, K. (2011). Application of Sinc-collocation method for solving a class of nonlinear Fredholm integral equations. Journal of Computers and Mathematics with Applications, 62, 3292-3303.

[4] Rashidinia, J., \& Zarebnia, M. (2005). Numerical solution of linear integral equations by using Sinc collocation method. Journal of Applied Mathematics and Computation, 168, 806-822.

[5] Zarebnia, M. (2010). Sinc numerical solution for the Volterra integro-differential equation. Journal of Commun Nonlinear Sci Numer Simulat, 15,700-706.

[6] Okayama, T., Matsuo, T., \& Sugihara, M,. (2010). Sinc-collocation methods for weakly singular Fredholm integral equations of the second kind. Journal of Computational and Applied Mathematics, 234, 1211-1227.

[7] Adibi, H., \& Assari, P. (2010). Chebyshev wavelet method for numerical solution of Fredholm integral equation of the first kind. Journal of Math. Prob. Eng.

[8] Rashed, M. T. (2003). Expansion method to treat integral equations plus. Journal of Applied Mathematics and Computation ,135, 65-72.

[9] Baker, C. T. H. (1969). The Numerical Treatment Of Integral Equations. Oxford. Clarendon Press.

[10] Kress, R. (1998). Linear Integral Equation. New York: Springer-Verlag.

[11] Stenger, F. (1993). Numerical Methods Based On Sinc And Analytic Functions. New York: Springer.

[12] Sugihara, M, \& Matsuo, T. (2004). Recent developments of the Sinc numerical methods. Journal of Comput Appl Math, 673-689.

[13] Sugihara, M.( 2003). Near optimality of the Sinc approximation. Journal of Math Comput, 72, 768-786.

[14] Prem, K. K., \& Pratap, P.( 2002). Computational Methods for Linear Integral Equations. Springer.

[15] Babolian, E., Lotfi, T., \& Paripour, M. (2007). Wavelet moment method for solving Fredholm integral equations of the first kind. Journal of Applied Mathematics and Computation, 186, 1467-1471. 


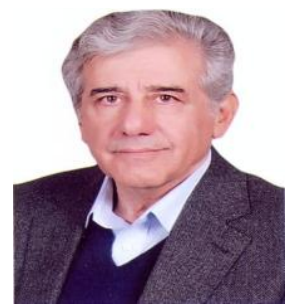

Khosrow Maleknejad obtained his MSc degree in applied mathematics from Tehran University, Iran, in 1972 and received his $\mathrm{PhD}$ degree in applied mathematics in numerical analysis area from the University of Wales, Aberystwyth, UK in 1980. In September 1972, he joined the School of Mathematics at Iran University of Science and Technology(IUST), Narmak, Tehran, Iran where he was previously lecturer, assistant professor and associate professor at IUST. He has been a professor since 2002 at IUST. He was a visiting professor at the University of California at Los Angeles in 1990. His research interests include numerical analysis in solving ill-posed problems and solving Fredholm and Volterra integral equations. He has authored as a Editor-in-chief of the International Journal of Mathematical Sciences, which publishers by Springer.He is a member of the AMS.

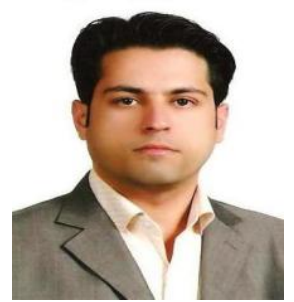

Yaser Rostami obtained his MSc degree in mathematics from the Islamic Azad University, Karaj Branch, Iran (KIAU) in 2007, He is a PhD degree student in applied mathematics in numerical analysis area from the KIAU. In 2008, he joined the Faculty of the Department Basic Science at Islamic Azad University, Malard Branch, Iran (IAUMALARD) where he was previously lecturer at IAUMALARD. His research interests include numerical analysis in solving Integral Equations and Wavelets. He has authored of several books of Calculus and Differential Equation. He is a member of the IMS.

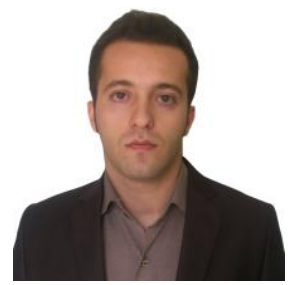

Hamed Shahi Kalalagh obtained his MSc degree in applied Mathematics from Iran University of Science and Technology, Narmak, Tehran, Iran(IUST) in 2012, and also he is a PhD degree student in applied mathematics in numerical analysis area from the IUST. His research interests include numerical analysis in solving integral equations and Wavelets. 